\title{
DESIGN AND HIGH POWER PROCESSING OF RFQ INPUT POWER COUPLERS*
}

\author{
Y. W. Kang, A. Vassioutchenko, A. V. Aleksandrov, D. Anderson, M. S. Champion, M. Crofford, \\ P. Gibson, T. Hardek, P. Ladd, M. P. McCarthy, D. Stout \\ Oak Ridge National Laboratory (ORNL), Oak Ridge, TN, U.S.A
}

\begin{abstract}
A RF power coupling system has been developed for future upgrade of input coupling of the RFQ in the SNS linac. The design employs two coaxial loop couplers for 402.5 MHz operation. Each loop is fed through a coaxial ceramic window that is connected to an output of a magic- $T$ waveguide hybrid through a coaxial to waveguide transition. The coaxial loop couplers are designed, manufactured, and high power processed. Two couplers will be used in parallel to power the accelerating structure with up to total $800 \mathrm{~kW}$ peak power at $6 \%$ duty cycle. RF and mechanical properties of the couplers are discussed. Result of high power RF conditioning that is performed in the RF test facility of the SNS is presented.
\end{abstract}

\section{INTRODUCTION}

Various RF coupling methods were used on RFQ structures [1][2]. A coaxial power coupling system for 402.5 MHz RFQ has been developed at SNS for full power operation in the future. The new coupling system developed and shown in this paper employs two loop couplers through a hybrid power splitter to feed the structure in parallel. Figure 1 shows the connection using a waveguide magic-T to deliver equal power to the loops with good isolation between the ports. The coaxial alumina ceramic window employed in this design is identical to the 81 coupler windows presently being used on the SNS SRF cavities operating at $805 \mathrm{MHz}$. [3][4]. The SRF couplers with electrically coupling antenna were successfully conditioned and tested for forward power of $650 \mathrm{~kW}$ peak, $8 \%$ duty cycle at $805 \mathrm{MHz}$. Operation of linac with the windows has shown excellent RF power handling capability and mechanical reliability.

The plan was to use the existing RFQ accelerating structure with no modification; the same coupler end dimensions of the coupler are needed. Using coaxial ceramic windows with a $10 \mathrm{~cm}$ diameter that is much greater than the $4 \mathrm{~cm}$ coupling port diameter requires a tapered coaxial transmission line section in the vacuum side of the coupler assembly. The RFQ in the SNS linac operates at $402.5 \mathrm{MHz}$ with about $700 \mathrm{~kW}$ peak, $6 \%$ duty cycle pulsed RF power. Each coupler needs to be conditioned to about $400 \mathrm{~kW}$. Modification of the inner and outer conductors was needed for impedance matching between the ceramic window and the loop. In the following, design, manufacturing, and RF power testing of the couplers are described.

*This work was supported by SNS through UT-Battelle, LLC, under contract DE-AC05-00OR22725 for the U.S. DOE.

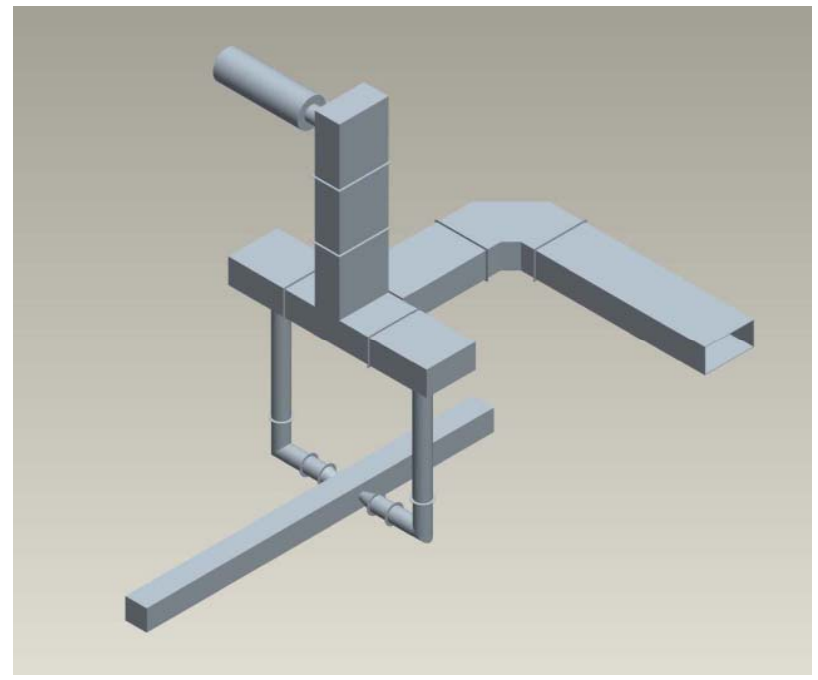

Figure 1: RF power coupling with two coaxial loop couplers to an RFQ structure. Magic-T waveguide power splitter is used to have isolation between the two ports.

\section{DESIGN AND MANUFACTURING}

Simulation and preliminary measurements showed that the window design can work well at around $400 \mathrm{MHz}$. Computer simulation was made to evaluate designs for the coupler consists of ceramic window, tapered transition, and coupling loop that resulted return loss $>30$ $\mathrm{dB}$ and insertion loss $<0.05 \mathrm{~dB}$ at $402.5 \mathrm{MHz}$. The RF heating on the center conductor was estimated to be 0.37 $\mathrm{W}$ for $1 \mathrm{~kW}$ average input power. Outside conductor that is dissipating only $0.15 \mathrm{~W}$ for $1 \mathrm{~kW}$ average input power requires light cooling since the outer conductor is connected to the temperature controlled cavity body. The complete processing setup was also simulated with two couplers on a bridge waveguide.

Figure 2 shows the design of the coupler assembly. The center conductor of the loop antenna is built as a single piece with the center conductor of the ceramic window. A small water cooled copper piece connects the center conductor and the outer conductor at the end of the reduced diameter coaxial section to form a complete loop antenna. The loop carries an $1 / 8$ " cooling tube circuit that provides a flow of water at $1 \mathrm{gpm}$ through the outer conductor to cool the center conductor.

Two vacuum ports with 2-3/4" flanges placed directly in front of the ceramic window on the vacuum-side have RF shielding with axial slots on the outer conductor of the coaxial transmission line. 


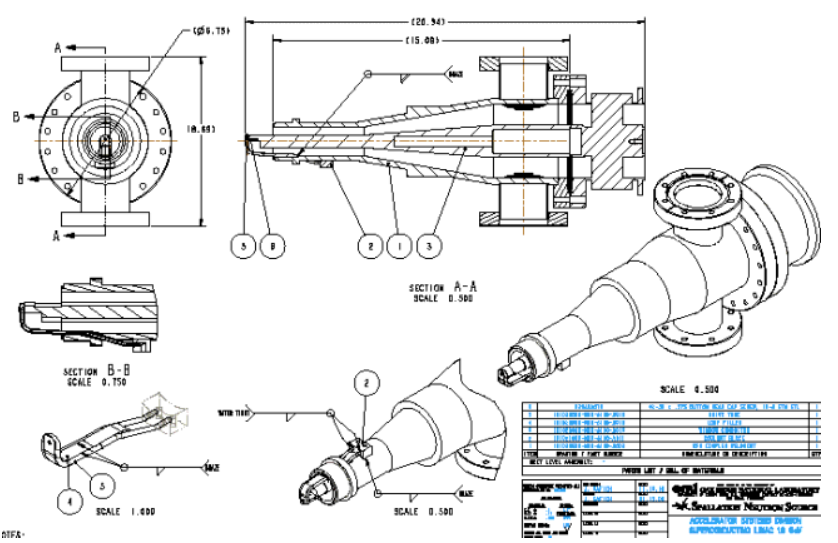

Figure 2: Coaxial loop coupler assembly.

A coaxial transmission system has been chosen for connecting the couplers between the klystron output waveguide and the RFQ. The coaxial loop antenna has coupling area enough to have slightly more than critical coupling to the cavity and port dimensions are fixed for installation on the cavity with no change. A 4-1/16" to 61/8" EIA coaxial transition and a waveguide to coaxial transformer with a 6-1/8" EIA coaxial port are used to connect to the air-side coaxial flange of the window. A short 4-1/16" coaxial section is modified for precise matching to the $100 \mathrm{~mm}$ ceramic window flange.

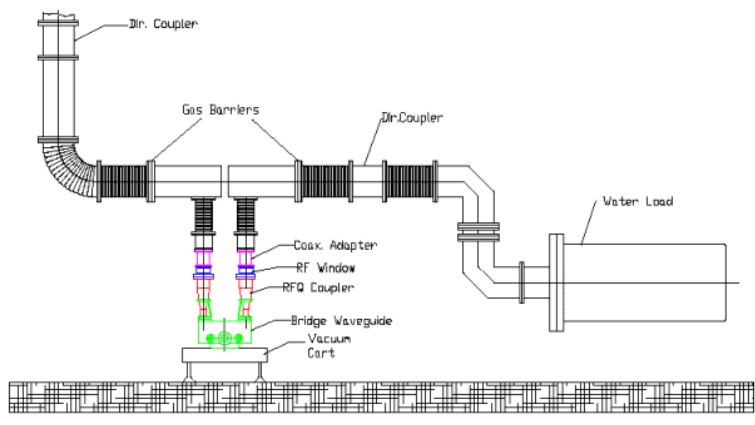

Figure 3: Coupler conditioning setup with two couplers connected back to back through the bridge waveguide.

Maintaining a good vacuum around the vacuum side of the window is critical for reliable operation of the coupler system. Two vacuum ports are placed in front of the ceramic window for best protection of the windows. Either one or both of the ports can be used for efficient vacuum pumping.

Figure 3 shows the coupler conditioning setup with two couplers connected back to back through a bridge waveguide. WR-2100 waveguide run is transformed to 6$1 / 8$ " coaxial system to feed the two couplers on the bridge waveguide and then transformed back to rectangular waveguide that is terminated with a matched water load at the end. Exact hardware identical to the hardware used for installation to cavity is used for the couplers connecting to the bridge waveguide.

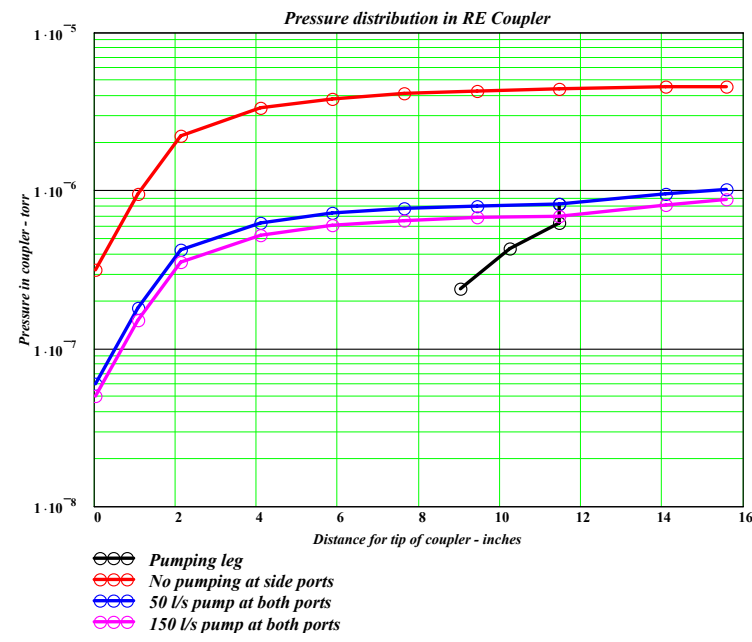

Figure 4: Vacuum pressure distribution in the coupler.

Figure 4 shows the pressure distribution along the coupler with and without pumping at the side pumping ports with out-gassing rates of $1 * 10^{-10} \mathrm{~T}-1 / \mathrm{s}-\mathrm{cm}^{2}$ for the coupler body and $1 * 10^{-7} \mathrm{~T}-1 / \mathrm{s}-\mathrm{cm}^{2}$ for the window being assumed. The pressure distribution in the coupler was analyzed using a multi node model with 10 nodes distributed along the length of the coupler and two additional modes for each of the two 3-1/8" inch pumping ports located at approximately $3 / 4$ of the coupler's depth. The model assumed that the pumping provided from the RFQ was infinite but limited by the inlet conductance to the coupler.

\section{TESTING AND RF PROCESSING}

All vacuum side parts were successfully manufactured and delivered. The bridge waveguide made of stainless steel was successfully manufactured and prepared. High power testing and conditioning started after cleaning and assembling the parts to construct complete setup for coupler processing. Figure 5 shows the RF response of the bridge waveguide with two couplers connected back to back as shown in Figure 3.

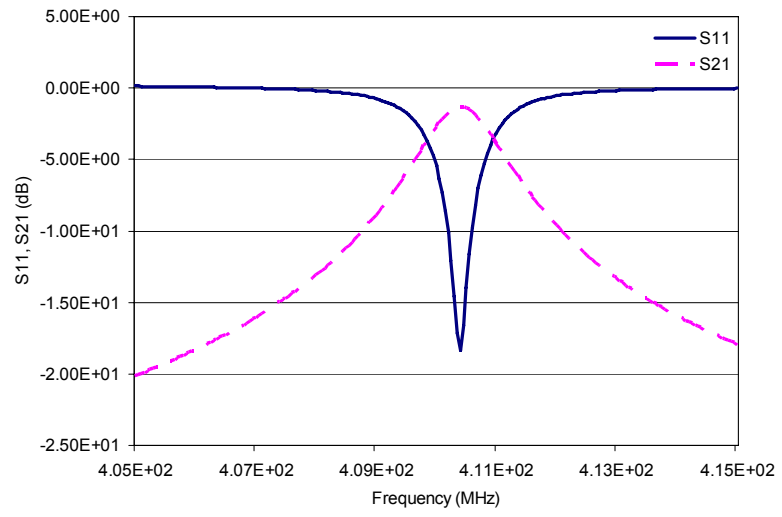

Figure 5: Measured RF response of two couplers connected back to back through the bridge waveguide. 
Figure 6 shows the setup for RF processing in the RF test facility with complete vacuum circuit. Waveguide to coaxial transitions are used to connect to the klystron at the input and the water load at the output.

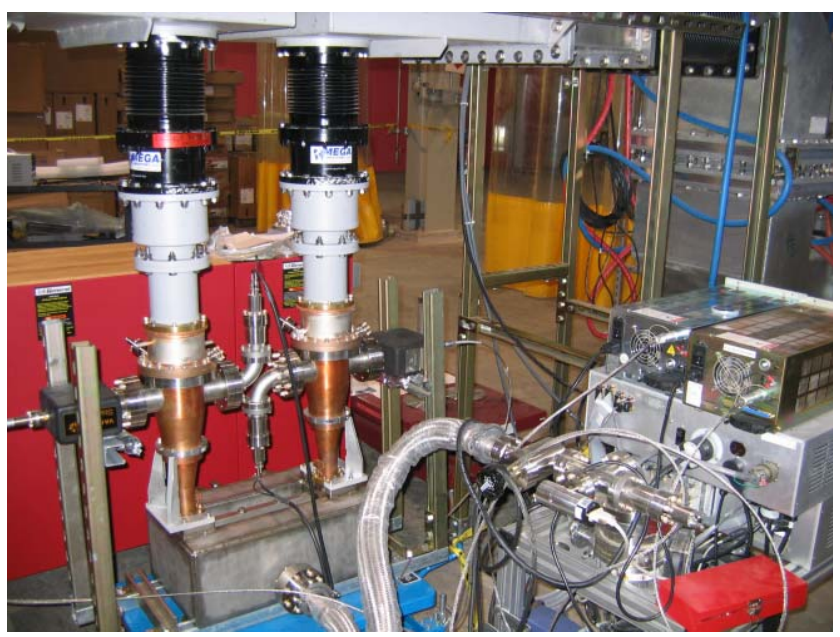

Figure 6: Couplers are mounted on the bridge waveguide for RF processing.

The bridge waveguide has an additional tuning mechanism that can deliver $\pm 3 \mathrm{MHz}$ tuning range. Initially, the resonant frequency was higher but the frequency was brought down to the right frequency by adjusting the gap between the bridge and the waveguide bottom along with the vacuum pumping that added even more shift in the frequency.

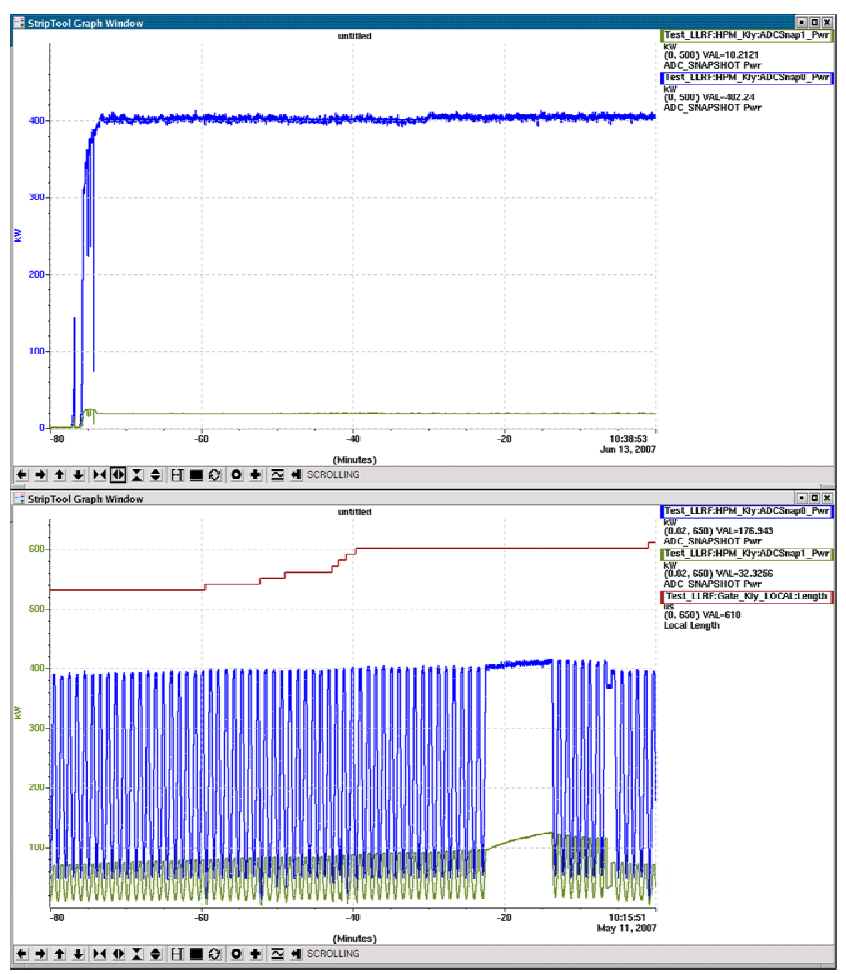

Figure 7: RF power deposited to the couplers during RF conditioning.
Figure 7 shows the power deposited to the couplers during the high power conditioning. RF processing was done with a short pulse $(\sim 0.1 \mathrm{msec})$ at 10 PPS to reach $400 \mathrm{~kW}$, the intended maximum power, and then the pulse width and the repetition rate were slowly increased to 1.0 msec and 60 PPS, respectively. When the full processing level was reached with constant power, the power was cycled between near zero and the peak power [4].

\section{DISCUSSION AND CONCLUSION}

The goal of this work was to construct an RF coupling system that is simpler and more robust for full beam power operation than the existing RFQ accelerating structure. Higher power rated couplers with proven electrical and mechanical reliability can improve system availability. Moving the couplers further down-stream and with a better vacuum system can improve the cavity vacuum and protection of the coupler windows. High power RF processing of the system has been performed successfully in the RF test facility at the SNS using 2.5 MW, $402.5 \mathrm{MHz}$ klystron system that can run up to $8 \%$ duty cycle.

Each coupler needs to over-couple the bridge waveguide when two couplers are used in series. Vacuum and the electron current were monitored while the vacuum was directly used for inhibiting the RF power input to the klystron as an interlock. An arc detector and an electron probe were used near the window to monitor the vacuum activity and to interlock the RF with vacuum pressure to protect the ceramic window.

\section{ACKOWLEDGEMENT}

The authors thank Robert Peglow, Chris Luck, John Crandall, and Debra Douglas of SNS project for their support in various tasks in preparing the couplers and the RF systems for processing. Also thanks are due to the mechanical and electrical groups of the SNS Project who provided the successful operation of the facility.

\section{REFERENCES}

[1] J. W. Staples, "RFQs - An Introduction," AIP Conference Proceedings, No. 249, Vol. 2

[2] D. Schrage et al, "CW RFQ Fabrication and Engineering," International LINAC Conference 1998, Argonne, IL, August 1998

[3] Y. Kang et al., "Electromagnetic Simulations and Properties of the Fundamental Power Couplers for the SNS Superconducting Cavities," PAC2001, Chicago, IL, June 2001

[4] M. Stirbet et al, "RF Conditioning And Testing Of Fundamental Power Couplers for SNS Superconducting Cavity Production," PAC2005, Knoxville, TN, June 2005 